\title{
Centre for health information quality
}

\author{
Derek Richards \\ Director, Centre for Evidence-based Dentistry, Oxford, UK
}

\author{
The Centre for Health Information Quality (CHIQ; www.hfht.org/chiq), was \\ established in 1997 by the UK government's Department of Health. CHIQ acts as a \\ clearing house for all aspects of patient information, providing practical advice to \\ the UK National Health Service (NHS) and others about the production of good \\ quality information for patients. \\ Evidence-Based Dentistry (2004) 5, 108-109. doi:10.1038/sj.ebd.6400289
}

CHIQ was initially established in 1997 through the NHS Executive in response to the 1996 Patient Partnership Strategy which recommended building a central resource to facilitate production of patient information by:

- building links between patient groups and organisations producing effectivenessand outcomes-information, to ensure that producers of information are aware of patients' priorities;

- identifying and facilitating the production of "effectiveness information" in a form helpful to individual patients;

- acting as an expert resource for NHS staff and patient representative groups seeking to produce or procure high-quality patient information resources.
In the following year the Information for Health strategy recommended strengthening the role of CHIQ, enabling it to play a stronger lead in accrediting patient and public information. The stated intention was to develop CHIQ's capacity to achieve a greater impact on health and health services provided by the NHS. CHIQ was also to have a voice in the assessment and accreditation of on-line information resources within the National Electronic Library.

CHIQ has been involved in the development and appraisal of content for the Conditions and Treatments Database of NHS Direct Online, and works with the UK National Institute of Clinical Excellence to develop patient versions

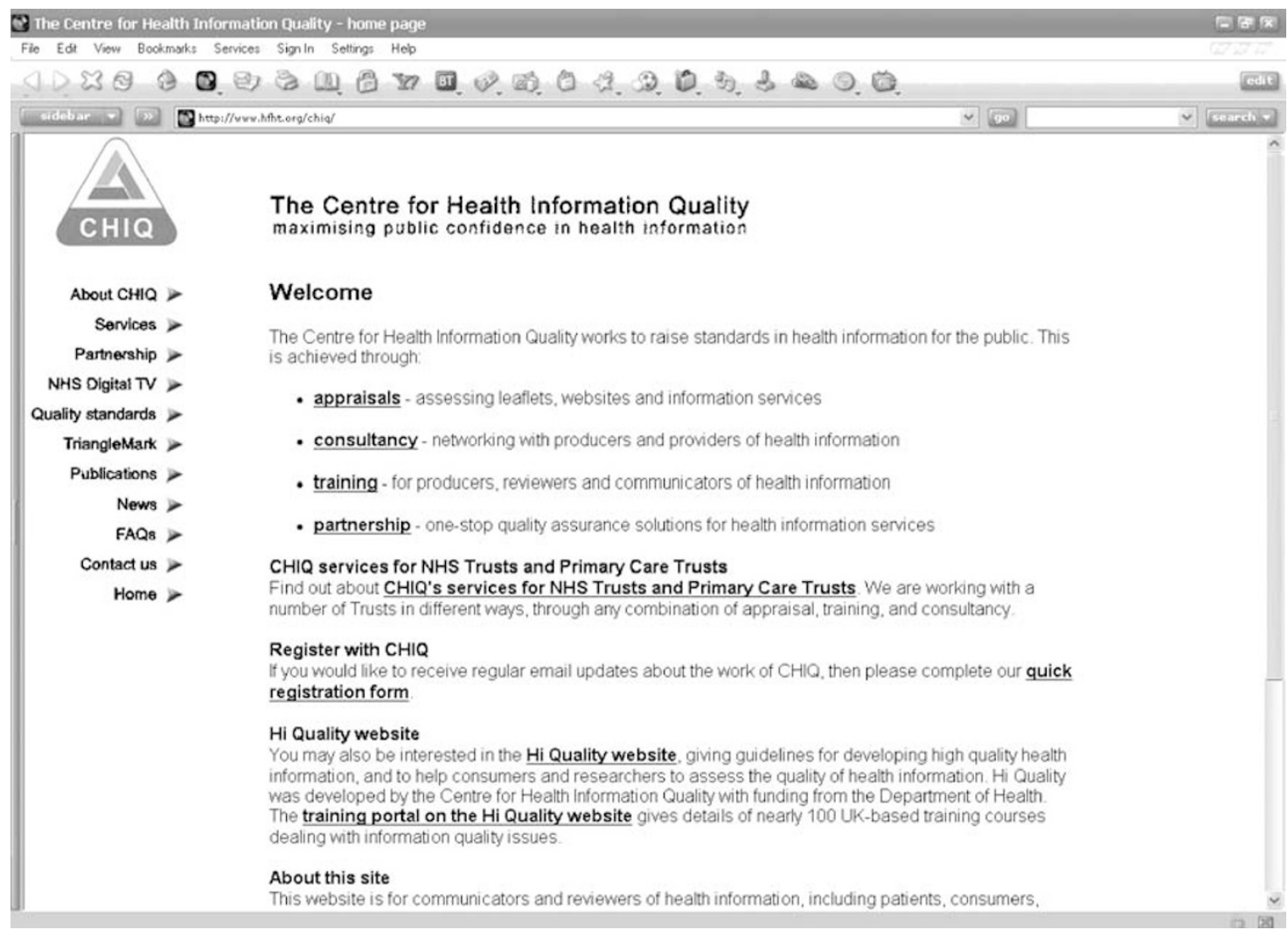

Figure 1. Centre for Health Information Quality homepage. 


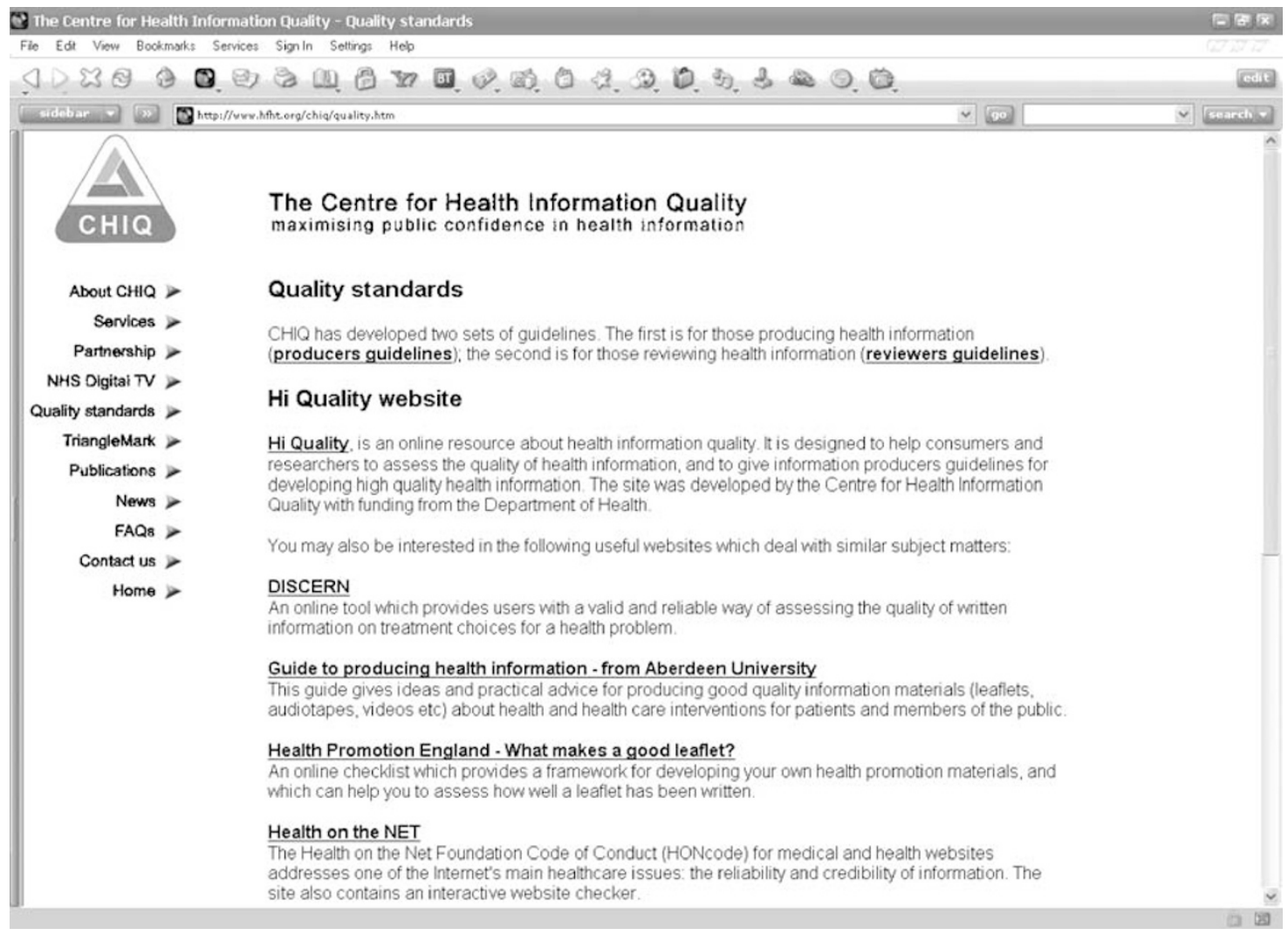

Figure 2. Centre for Health Information Quality Standards.

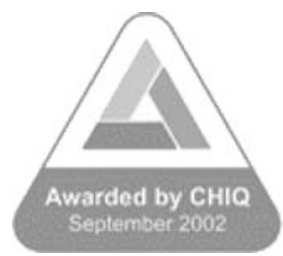

Figure 3. The CHIQ TriangleMark.

of their clinical guidelines. It has also been participating in a range of other initiatives, which are detailed on their website.

CHIQ is based in Winchester at the Help for Health Trust (HFHT), an independent charity providing health information databases (www.hfht.org). The homepage of their website (Figure 1) provides a brief history of CHIQ and a list of different organisations with whom they have worked.
The website is simple, clear and uncluttered, with links to the main pages on the customary left side of the page. Here you will also find links to services provided by CHIQ and their various partners. For example, CHIQ has recently completed a quality assurance programme across all public NHS Digital TV pilot services: NHS Digital TV started its roll-out during the summer of 2004 and will be providing information on:

- NHS services (such as directories of general practitioners, dentists, pharmacies, etc).

- An encyclopaedia of illnesses and conditions, tests, treatments and operations.

- Self-care advice on treating common health problems.

- Advice on healthy living.

- Hot topics on current health issues.

The Quality Standards link takes you to a page containing a wide range of useful information for producing and assessing health information (Figure 2), including producers' and reviewers' guidelines produced by CHIQ.

CHIQ has also developed a TriangleMark (Figure 3) to help reassure the public about the quality of health information. The TriangleMark can only be displayed when the information meets CHIQ standards.

The triangle has been chosen to represent three themes of quality:

- Accurate - The information is up-todate and sources of evidence are made clear. - Clear - The information is clearly communicated.

- Relevant - The information has been developed in association with the consumers.

So if the health information you are reading does not have a TriangleMark, you are in the process of developing a new leaflet, or you are assessing the quality of some health information you have been given, the CHIQ site is worth a visit. 